\title{
Strain-oriented strategy for guiding cardioprotection initiation of breast cancer patients experiencing cardiac dysfunction
}

\author{
Ciro Santoro ${ }^{1}$, Roberta Esposito ${ }^{1,2}$, Maria Lembo', Regina Sorrentino ${ }^{1}$, \\ Irene De Santo ${ }^{3}$, Federica Luciano', Ofelia Casciano', Mario Giuliano ${ }^{3}$, \\ Sabino De Placido ${ }^{3}$, Bruno Trimarco ${ }^{1}$, Patrizio Lancellotti ${ }^{4,5}$, Grazia Arpino ${ }^{3}$, and \\ Maurizio Galderisi $\mathbb{1}^{1}{ }^{1 *}$
}

${ }^{1}$ Department of Advanced Biomedical Science, Federico II University Hospital Naples, Via Pansini 5, 80131Naples, Italy; ${ }^{2}$ Mediterranea Cardio Centro, Naples, Via Orazio 2 , 80122, Italy; ${ }^{3}$ Department of Clinical Medicine, Federico II University Hospital Naples, Naples, Italy; ${ }^{4}$ University of Liège Hospital, GIGA Cardiovascular Sciences, Liège, Belgium; and ${ }^{5}$ Gruppo Villa Maria Care and Research, Anthea Hospital, Bari, Italy

Received 22 February 2019; editorial decision 29 June 2019; accepted 3 July 2019; online publish-ahead-of-print 21 July 2019

\begin{abstract}
Aims
This study assessed the impact of the strain-guided therapeutic approach on cancer therapy-related cardiac dysfunction (CTRCD) and rate of cancer therapy (CT) interruption in breast cancer.

Methods

and results

We enrolled 116 consecutive female patients with HER2-positive breast cancer undergoing a standard protocol by EC (epirubicine + cyclophosphamide) followed by paclitaxel + trastuzumab (TRZ). Coronary artery, valvular and congenital heart disease, heart failure, primary cardiomyopathies, permanent or persistent atrial fibrillation, and inadequate echo-imaging were exclusion criteria. Patients underwent an echo-Doppler exam with determination of ejection fraction (EF) and global longitudinal strain (GLS) at baseline and every 3 months during CT. All patients developing subclinical (GLS drop $>15 \%$ ) or overt CTRCD (EF reduction $<50 \%$ ) initiated cardiac treatment (ramipril+ carvedilol). In the $99.1 \%$ (115/116) of patients successfully completing CT, GLS and EF were significantly reduced and $E / e^{\prime}$ ratio increased at therapy completion. Combined subclinical and overt $C T R C D$ was diagnosed in 27 patients (23.3\%), 8 at the end of EC and 19 during TRZ courses. Of these, 4 (3.4\%) developed subsequent overt CTRCD and interrupted CT. By cardiac treatment, complete EF recovery was observed in two of these patients and partial recovery in one. These patients with EF recovery re-started and successfully completed CT. The remaining patient, not showing EF increase, permanently stopped $\mathrm{CT}$. The other 23 patients with subclinical CTRCD continued and completed CT.
\end{abstract}

Conclusion These findings highlight the usefulness of 'strain oriented' approach in reducing the rate of overt CTRCD and CT interruption by a timely cardioprotective treatment initiation.

Keywords breast cancer - cancer therapeutics related cardiac dysfunction $\quad$ global longitudinal strain

- ejection fraction - heart failure

\section{Introduction}

Nowadays, cancer therapy (CT) progressively prolongs survival in oncologic diseases. Nevertheless, it may expose patients to lifethreatening complications involving cardiovascular system. Thus, cardiotoxicity may become one of the main determinants of quality of life impairment and mortality in this specific population. ${ }^{1,2}$
Anti-cancer drugs can lead to several adverse cardiovascular effects, such as arterial hypertension, myocardial ischaemia, thromboembolic complications, arrhythmias, and conduction disturbances. ${ }^{3}$ However, the most frequent and clinically relevant form of cardiotoxicity corresponds to a dilated and hypokinetic cardiomyopathy leading to overt heart failure $(\mathrm{HF}) .{ }^{4}$ Cancer therapy-related cardiac dysfunction (CTRCD) can be due to different kinds of treatment: anthracyclines 
provoke a dose-cumulative myocardial damage with irreversible cellular necrosis (Type I cardiotoxicity), while other agents, such as trastuzumab (TRZ), lead to a non-dose-related but reversible cardiac impairment (Type II cardiotoxicity). ${ }^{5-7}$ The sequential or concurrent use of these two different types of agents may increase myocardial injury and CTRCD is often the result of the combined detrimental effect of the two therapies. ${ }^{7}$

A timely initiation of cardioprotective treatment for CTRCD is pivotal to continue the ongoing CT till completion and reduce the risk of overt HF. Cardiac treatment of CTRCD is currently guided by 2D echocardiographic evaluation of left ventricular (LV) ejection fraction (EF). ${ }^{8}$ However, EF is affected by several limitations including its load-dependence, the need of geometric assumption for its calculation and, above-all, its substantial biological (day-to-day) variability that makes subtle changes often doubtful and questionable. ${ }^{9}$ As an alternative, global longitudinal strain (GLS), easily obtainable by speckle tracking echocardiography, has shown optimal feasibility and temporal reproducibility and its changes may precede EF reduction in the general population and in oncologic patients as well. ${ }^{10}$

The lack of definite results on clinical outcomes produced by a GLS-oriented strategy, i.e. the impact of a strain-guided cardioprotective therapy initiation on the development of overt HF, makes this innovative approach not ready yet for addressing the decision-making in this clinical setting. Accordingly, the present study was designed to assess the impact of the strain-oriented therapeutic approach in reducing the development of overt CTRCD and the rate of CT interruption in breast cancer patients.

\section{Methods}

\section{Study population}

After their informed consent was collected, 116 consecutive female patients with diagnosis of HER2-positive breast cancer were enrolled in the Oncologic Clinic of Federico II University Hospital. The approval by the Ethical Committee of Federico II University Hospital was obtained. Patients with coronary artery disease, overt HF, primary cardiomyopathies, haemodynamically significant valvular heart disease, permanent or persistent atrial fibrillation, congenital heart disease, and inadequate echocardiographic imaging before starting CT therapy were excluded. The presence of cardiovascular risk factors including arterial hypertension, diabetes mellitus, dyslipidaemia, and smoke habit was collected in individual patients.

All patients underwent a standard transthoracic echo-Doppler study at baseline before the start of CT and every 3 months during the therapy. Blood pressure was measured in supine position by a cuff sphygmomanometer (average of three measurements) at the end of each exam.

\section{Cancer therapy protocol}

Patients underwent a CT standard protocol defined as EC [anthracycline epirubicine $\left(90 \mathrm{mg} / \mathrm{m}^{2}\right)+$ cyclophosphamide $\left.\left(600 \mathrm{mg} / \mathrm{m}^{2}\right)\right]$ for four cycles, with an inter-cycle interval of 21 days. At the end of EC, CT continued with the administration of paclitaxel $\left(80 \mathrm{mg} / \mathrm{m}^{2}\right)$ in combination with TRZ ( $4 \mathrm{mg} / \mathrm{kg}$ for the first week and then $2 \mathrm{mg} / \mathrm{kg}$ weekly for 11 cycles), followed by paclitaxel $\left(80 \mathrm{mg} / \mathrm{m}^{2}\right)+$ TRZ $(6 \mathrm{mg} / \mathrm{kg})$ for 13 cycles (inter-cycle pauses of 21 days).

A complete echo-Doppler exam was performed at baseline, after EC completion, and every 3 months during and at the end of the remaining CT. If overt CTRCD was suspected (decrease in EF $>10$ percentage points, to a value below $50 \%$ according to the 2016 American Society of Echocardiography (ASE)/European Association of Cardiovascular Imaging (EACVI) Expert Consensus on the role of cardiac imaging in adult cancer patients), ${ }^{5}$ a repeated echocardiographic exam was performed within 2-3 weeks to confirm the finding. When two consecutives evaluation showed overt CTRCD, we referred to the oncologist to determine whether discontinuation of anti-cancer drugs was needed (i.e. in presence of cardiac symptoms), as recommended. ${ }^{8}$ Of those who interrupted the anti-cancer drug administration, only the ones with full recovery (EF recovery to baseline values) or partial recovery (EF recovery above $50 \%$ but below baseline values) re-started the planned CT protocol. Patients with a GLS reduction $>15 \%$ from baseline, independently on EF values, were diagnosed to have a subclinical CTRCD. ${ }^{5}$ Noteworthy, in our established protocol all patients developing CTRCD (either overt or subclinical) initiated a cardioprotective treatment with angiotensinconverting enzyme (ACE)-inhibitors (ramipril) and beta-blockers (carvedilol), at a maximal tolerated dosage, as recommended. ${ }^{8}$

\section{Echo Doppler exam}

Standard echo-Doppler exam including speckle tracking echocardiography of apical views were performed by a Vivid E9 ultrasound machine (GE Healthcare, Horten, Norway), using a $2.5 \mathrm{MHz}$ transducer with harmonic capability.

Two-dimensional and Doppler exams were performed according to the standards of our laboratory. ${ }^{11,12}$ LV analysis was quantified in agreement of 2015 recommendations of the ASE and the EACVI ${ }^{13}$ and the EACVI standardization of the echo report. ${ }^{14}$ Two-dimensional echo derived EF was calculated from LV end-diastolic and end-systolic volumes (from apical four- and two-chamber views) computed by the modified Simpson rule. Relative wall thickness and LV mass were obtained by 2D guided M-mode imaging or directly from 2D longitudinal long-axis view. LV mass was indexed for height powered to $2.7 .^{15}$ Left atrial volume was indexed for body surface area. ${ }^{16}$ Transmitral Doppler inflow and pulsed Tissue Doppler were recorded in apical four-chamber view to analyse LV filling pattern (transmitral E/A ratio and $E$ velocity deceleration time), and the average of early diastolic velocities $\left(e^{\prime}\right)$ of septal and lateral mitral annulus was used to calculate the E/e' ratio. ${ }^{16}$

Speckle tracking echocardiography procedures were performed according to the standards of our laboratory. ${ }^{11,12}$ LV longitudinal deformation was recorded on 2D images of three consecutive cardiac cycles from the three apical (long-axis, four- and two-chamber) views at an approximately equal heart rate. An interactive software of a dedicated workstation (Echopac BT13 version, GE) allowed to automatically trace the endocardial-cavity interface, with possible, subsequent manual adjustment, and rejection of segments of poor imaging quality. Each of the three apical images was automatically divided into six myocardial segments. Peak negative longitudinal strain was measured from six segments in each of the three apical views and GLS was computed as the average of individual peak strain before aortic valve closure. Reproducibility of speckle tracking echo in our laboratory has been previously reported. ${ }^{17}$ GLS was considered in absolute value according to Chamber Quantification recommendations. ${ }^{13}$

\section{Statistical analysis}

Statistical analysis was performed by SPSS package, release12 (SPSS Inc., Chicago, IL, USA). Data are presented as mean value \pm standard deviation. Descriptive statistics were obtained by one factor ANOVA (Bonferroni post hoc intergroup analysis) and $\chi^{2}$ distribution with computation of exact $P$-value by Monte Carlo method and inter-group post hoc (Bonferroni test) comparison. The null hypothesis was rejected at twotailed $P<0.05$. 


\section{Results}

Figure 1 summarizes the behaviour of our overall breast cancer population through the entire study duration. The characteristics of the study population are shown in Table 1. In the overall population, $27.6 \%$ patients $(n=32)$ were hypertensive ( 22 treated with antihypertensive drugs including ACE-inhibitors, angiotensin II receptor blockers, beta-blockers, and calcium channel antagonists), and $19.8 \%$ $(n=23)$ overweight.

During the follow-up, no patient had an episode of atrial fibrillation, whereas effort dyspnoea was observed in four patients during TRZ treatment. The $99.1 \%(115 / 116)$ of the patients successfully completed CT. In this group, GLS $(P<0.0001)$ and EF $(P<0.002)$ were both reduced and $E / \mathrm{e}^{\prime}$ ratio increased $(P=0.028)$ at TRZ end in comparison with baseline (Table 2).

Combined subclinical and overt CTRCD was diagnosed in 27 of the 116 patients (23.3\%) (Figure 1A). The individual characteristics of these 27 patients are summarized in Table 3. Among eight hypertensive patients, six received antihypertensive treatment at baseline (data not in table). By comparing baseline echo parameters of patients with and without CTRCD, no significant difference of EF was observed whereas GLS was slightly higher $(P<0.02)$ in CTRCD group. The prevalence of hypertensive patients assuming antihypertensive drugs was comparable between the two groups $(66.7 \%$ and $75 \%$ in the CTRCD and non-CTRCD groups, respectively, $P=0.66)$.

All 27 CTRCD patients started cardioprotective treatment with ramipril (average daily dose $7.8 \pm 2.5 \mathrm{mg}$ ) and carvedilol (average daily dose $10.3 \pm 3.1 \mathrm{mg}$ ), without referring any significant side effect. Four of these patients (3.4\%) subsequently developed overt CTRCD and, based on cardiac symptoms, i.e. effort dyspnoea, and EF reduction 'below 50\%' (in at least two repeated echo exams), interrupted CT at variable TRZ cycle timing, after oncologic consultation (Figure 1B).
Worthy of note, in all these patients GLS was reduced by $>15 \%$. One of these patients did not show any EF increase (in two repeated echo exams within 3 weeks and, subsequently, after 1 month) and, being still symptomatic for effort dyspnoea, definitely stopped CT after oncologic consultation; a complete EF recovery to baseline values induced by cardioprotective treatment was observed in two patients, and a partial recovery in the remaining one (Figure 1C). The three patients showing EF recovery had also a concomitant GLS recovery (Figure 2) and could re-start the programmed CT protocol till completion.

The remaining 23 patients (19.8\%) developed subclinical CTRCD (GLS drop >15\%), 8 at the end of EC and 19 during TRZ courses, and-thanks to cardiac treatment-continued and successfully completed the TRZ cycles, without any interruption because they did not progress towards overt CTRCD. Table 4 compares the echo data obtained at baseline, CTRCD onset and the end of CT of the 23 patients developing subclinical CTRCD. Both EF and GLS were reduced at CTRCD onset compared to baseline (both $P<0.0001$ ), whereas significantly improved at the time of $\mathrm{CT}$ completion in

Table I Clinical characteristics of the overall study population at baseline

\begin{tabular}{|c|c|c|}
\hline Variables & Mean \pm SD & Range \\
\hline Age (years) & $52.5 \pm 12.1$ & $24-77$ \\
\hline Body mass index $\left(\mathrm{kg} / \mathrm{m}^{2}\right)$ & $26.1 \pm 4.6$ & $16.8-40.1$ \\
\hline Systolic BP (mmHg) & $125.8 \pm 15.2$ & 90-170 \\
\hline Diastolic BP (mm/Hg) & $78.4 \pm 8.5$ & $50-110$ \\
\hline Heart rate (bpm) & $72.8 \pm 11.4$ & $50-109$ \\
\hline
\end{tabular}

$\mathrm{BP}$, blood pressure; SD, standard deviation

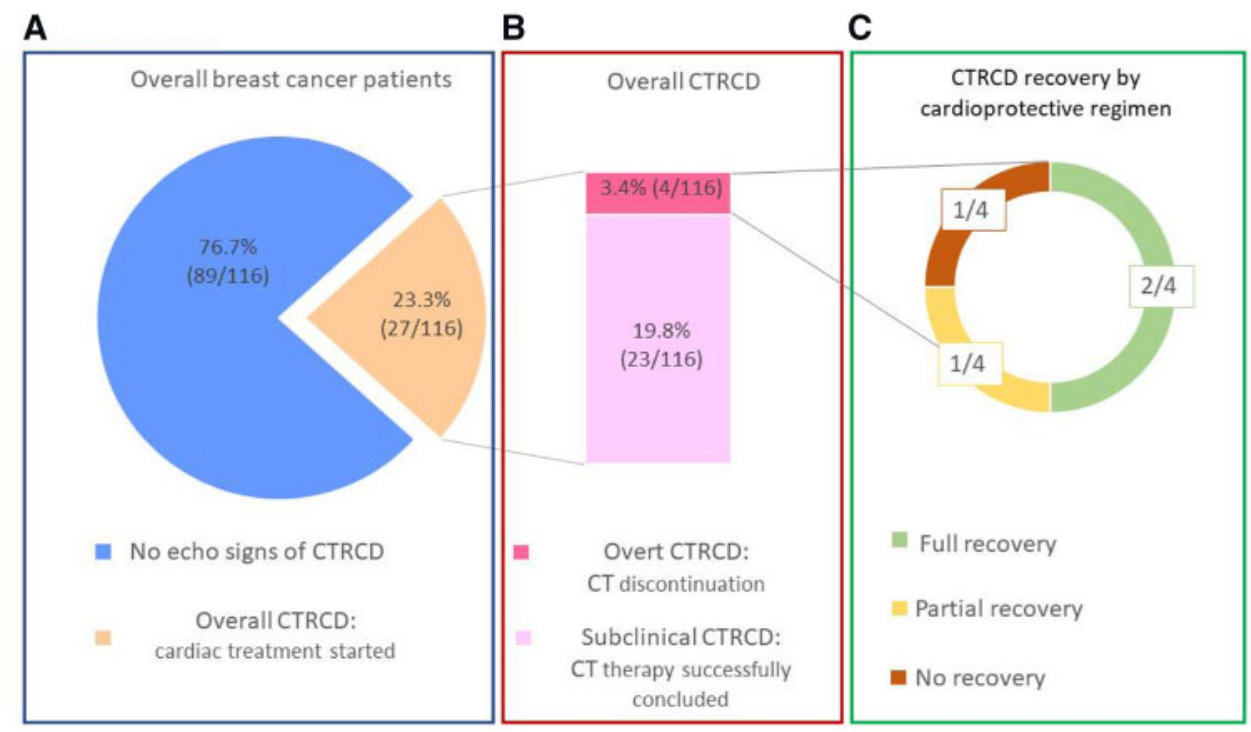

Figure I Picture of the study population. (A) Overall breast cancer population, (B) CTRCD (both subclinical and clinical) population, and (C) CTRCD recovery after cardioprotective regimen. CTRCD, cancer therapy-related cardiac dysfunction. 
Table 2 ANOVA comparison of echocardiographic parameters at baseline, at end EC, and at TRZ completion in patients without CTRCD $(n=89)$

\begin{tabular}{|c|c|c|c|}
\hline Variables & Baseline & End EC & End TRZ \\
\hline Relative wall thickness & $0.32 \pm 0.05$ & $0.32 \pm 0.06$ & $0.33 \pm 0.04$ \\
\hline $\mathrm{LV}$ mass index $\left(\mathrm{g} / \mathrm{m}^{2.7}\right)$ & $33.2 \pm 8.2$ & $34.1 \pm 8.2$ & $35.0 \pm 8.2$ \\
\hline LV EF (\%) & $63.4 \pm 3.9$ & $62.1 \pm 4.3$ & $61.4 \pm 4.0^{\mathrm{a}}$ \\
\hline LV GLS (\%) & $22.0 \pm 2.4$ & $21.0 \pm 2.5$ & $20.2 \pm 4.8^{b}$ \\
\hline Transmitral E/A ratio & $1.11 \pm 0.4$ & $1.10 \pm 0.3$ & $1.10 \pm 0.4$ \\
\hline E velocity DT (ms) & $210.1 \pm 44.4$ & $207.8 \pm 48.1$ & $211.8 \pm 46.0$ \\
\hline$E / e^{\prime}$ ratio & $7.5 \pm 2.4$ & $7.9 \pm 2.4$ & $8.9 \pm 2.7^{c}$ \\
\hline LAVi $\left(\mathrm{ml} / \mathrm{m}^{2}\right)$ & $26.4 \pm 6.6$ & $27.1 \pm 5.9$ & $26.1 \pm 6.0$ \\
\hline
\end{tabular}

CTRCD, cancer therapy-related cardiotoxicity; DT, deceleration time; EF, ejection fraction; End EC, end epirubicine + cyclophosphamide; End TRZ, end trastuzumab; GLS, global longitudinal strain; LAVi, left atrial volume index; LV, left ventricular.

${ }^{a} p<0.002$ between baseline and end of anticancer therapy.

${ }^{\mathrm{b}} P<0.0001$ between baseline and end of anticancer therapy

${ }^{c} P=0.028$ between baseline and end of anticancer therapy. comparison with both baseline and time of CTRCD onset. Figure 3 depicts a clinical case of a patient who developed subclinical CTRCD with a significant GLS drop, but successfully recovered both GLS and EF at the end of TRZ thanks to cardiac treatment.

\section{Discussion}

Our study demonstrates that in a population of HER2-positive breast cancer patients undergoing adjuvant therapy: (i) the use of GLS allows to identify subclinical CTRCD when EF is still normal, with a prevalence of $23.3 \%$ (27/116); (ii) all the 23 patients developing subclinical CTRCD without progression towards overt HF were able to complete CT without interruption, thanks to the help of cardioprotective treatment; (iii) among the four patients developing subsequent overt CTRCD, three could complete CT with an adequate cardioprotective treatment; (iv) in the subgroup of patients without CTRCD (76.7\%) a reduction in EF and GLS and an increase in E/e' ratio was detected at the end of $\mathrm{CT}$ in comparison with baseline.

Nowadays, the methodology for early diagnosing subclinical CTRCD in cancer patients remains controversial. Cardinale et al.

Table 3 Clinical characteristics of the patients developing subclinical and overt CTRCD

\begin{tabular}{|c|c|c|c|c|c|c|c|c|c|c|}
\hline Patient & Age & BMI & Smoke & HTN & Dyslipidaemia & DM 2 & $\begin{array}{l}\text { CTRCD } \\
\text { onset }\end{array}$ & $\begin{array}{l}\text { Baseline EF (\%) } \\
\text { and GLS (\%) }\end{array}$ & $\begin{array}{l}\text { CTRCD EF(\%) } \\
\text { and GLS (\%) }\end{array}$ & $\begin{array}{l}\text { End TRZ EF (\%) } \\
\text { and GLS (\%) }\end{array}$ \\
\hline 1 & 56 & 23.9 & 0 & 0 & 1 & 0 & $2 \mathrm{TRZ}$ & $60-23.4$ & 60-18.4 & $60-20.6$ \\
\hline 2 & 64 & 24.3 & 1 & 0 & 1 & 0 & $1 \mathrm{TRZ}$ & $63-25.1$ & 59-20.9 & 60-19.1 \\
\hline 3 & 54 & 24.8 & 1 & 1 & 0 & 0 & End EC & $60-28.4$ & $60-23.9$ & $68-25$ \\
\hline 4 & 68 & 35.2 & 0 & 0 & 1 & 0 & $9 \mathrm{TRZ}$ & $59-21.1$ & $60-17.2$ & $65-20.3$ \\
\hline 5 & 45 & 22.1 & 0 & 0 & 0 & 0 & End EC & $65-23$ & 67-19.0 & 59-17.8 \\
\hline 6 & 60 & 25.7 & 0 & 0 & 1 & 0 & End EC & $65-23$ & 60-19 & 64-17.8 \\
\hline 7 & 56 & 25.5 & 1 & 0 & 1 & 0 & End EC & $64-24.6$ & $58-20.5$ & 60-19.3 \\
\hline 8 & 24 & 24.3 & 1 & 1 & 0 & 0 & End EC & $69-24.8$ & $62-20.1$ & $66-22.1$ \\
\hline 9 & 47 & 19.1 & 0 & 0 & 1 & 0 & $1 \mathrm{TRZ}$ & $70-21.8$ & $57-17.5$ & $58-17.7$ \\
\hline 10 & 64 & 32.5 & 0 & 0 & 0 & 0 & End EC & $65-26.2$ & $65-20.9$ & $61-21.9$ \\
\hline 11 & 49 & 19.5 & 0 & 0 & 1 & 0 & End EC & $65-27.7$ & $68-22.5$ & 58-18.6 \\
\hline 12 & 48 & 27.8 & 1 & 0 & 1 & 0 & $1 \mathrm{TRZ}$ & $67-20.7$ & $65-16$ & $58-20.6$ \\
\hline 13 & 73 & 29.1 & 1 & 1 & 0 & 0 & End EC & $64-22.7$ & 59-18.7 & $58-22$ \\
\hline 14 & 46 & 22.3 & 0 & 0 & 1 & 0 & $1 \mathrm{TRZ}$ & $72-24.3$ & $62-20.4$ & 59-21.2 \\
\hline 15 & 73 & 31.2 & 0 & 1 & 1 & 0 & $3 \mathrm{TRZ}$ & $65-23.2$ & $48-14.3$ & \\
\hline 16 & 45 & 37.0 & 0 & 0 & 1 & 0 & $4 \mathrm{TRZ}$ & $67-23.4$ & $60-17.0$ & $61-22.7$ \\
\hline 17 & 28 & 21.0 & 0 & 0 & 0 & 0 & $6 \mathrm{TRZ}$ & $67-23.2$ & $58-16.7$ & $60-22.7$ \\
\hline 18 & 62 & 30.4 & 0 & 0 & 1 & 0 & $1 \mathrm{TRZ}$ & $58-20.0$ & $51-16.5$ & 60-18.0 \\
\hline 19 & 58 & 26.6 & 1 & 0 & 1 & 0 & $5 \mathrm{TRZ}$ & $60-21.4$ & 49-14.7 & \\
\hline 20 & 55 & 24.3 & 1 & 1 & 0 & 0 & $6 \mathrm{TRZ}$ & $65-27.1$ & $50-16.4$ & \\
\hline 21 & 65 & 23.2 & 0 & 0 & 1 & 0 & $6 \mathrm{TRZ}$ & $68-22.5$ & $52-17.3$ & $58-22.0$ \\
\hline 22 & 58 & 25.4 & 0 & 0 & 0 & 0 & $2 \mathrm{TRZ}$ & $65-20.0$ & $57-17.0$ & $64-23.2$ \\
\hline 23 & 46 & 21.6 & 1 & 1 & 1 & 0 & $9 \mathrm{TRZ}$ & 60-18.2 & $38-11.9$ & \\
\hline 24 & 54 & 24.5 & 1 & 0 & 0 & 0 & $1 \mathrm{TRZ}$ & 60-19.9 & $56-14.2$ & 60-18.9 \\
\hline 25 & 54 & 29.3 & 0 & 1 & 0 & 0 & $5 \mathrm{TRZ}$ & $62-19.6$ & 61-15.5 & 64-19.5 \\
\hline 26 & 62 & 32.0 & 0 & 0 & 1 & 1 & $11 \mathrm{TRZ}$ & $58-19.2$ & $53-15.1$ & 61-19.7 \\
\hline 27 & 50 & 27.8 & 0 & 1 & 0 & 0 & $8 \mathrm{TRZ}$ & $65-20.8$ & $57-17.5$ & 59-17.9 \\
\hline
\end{tabular}

BMI, body mass index; DM 2, diabetes mellitus type 2; EC, epirubicine + cyclophoshamide; EF, ejection fraction; GLS, global longitudinal strain; HTN, arterial hypertension; LV, left ventricular; TRZ, trastuzumab. 

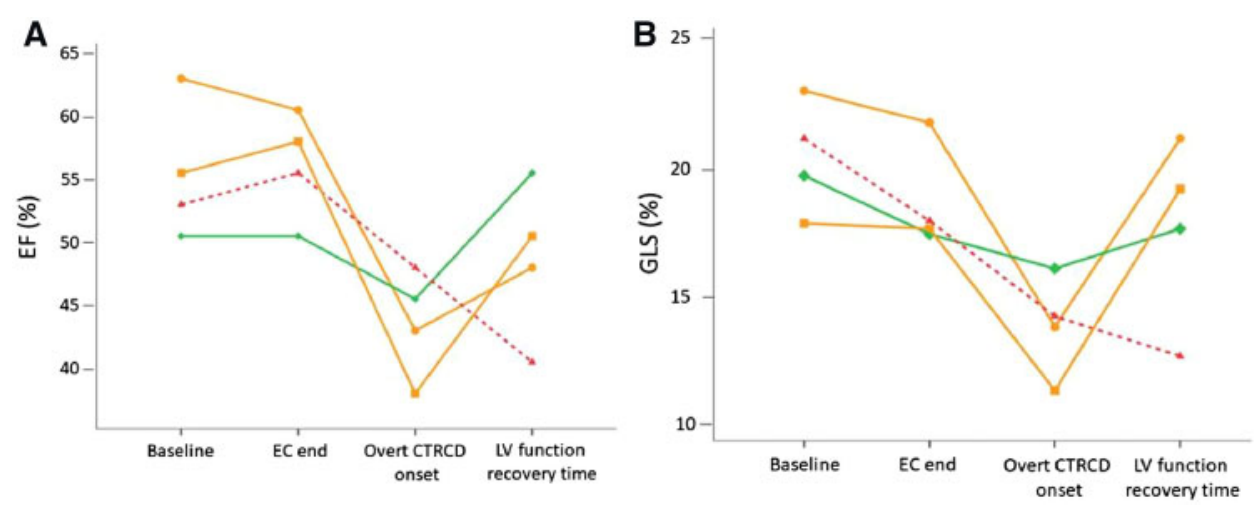

Figure 2 Behaviour of EF (A) and GLS (B) at EC end, at the time of overt CTRCD onset (during TRZ) and at the time of LV function recovery in the individual patients developing overt CTRCD. Solid green lines indicate patients with full LV function recovery thanks cardioprotective regimen (at 60 and 95 days, respectively); solid orange lines indicate patient with partial LV function recovery thanks cardioprotective regimen (at 120 days). Dotted red lines indicate patient without LV function recovery (after 51 days after interrupting CT) and forced to permanently stop CT. CTRCD, cancer therapy-related cardiac dysfunction; EC, epirubicine + cyclophosphamide; EF, ejection fraction; GLS, global longitudinal strain; LV, left ventricular.

Table 4 ANOVA comparison of echocardiographic parameters at baseline, at end EC, and at TRZ completion in patients with subclinical CTRCD $(n=23)$

\begin{tabular}{|c|c|c|c|}
\hline Variables & Baseline & End EC & End TRZ \\
\hline Relative wall thickness & $0.31 \pm 0.04$ & $0.29 \pm 0.04$ & $0.32 \pm 0.04$ \\
\hline LV mass index $\left(\mathrm{g} / \mathrm{m}^{2.7}\right)$ & $35.8 \pm 9.1$ & $40.2 \pm 8.3$ & $39.4 \pm 10.2$ \\
\hline LV EF (\%) & $62.8 \pm 3.5^{\mathrm{a}}$ & $53.0 \pm 6.0^{b}$ & $60.7 \pm 2.0^{c}$ \\
\hline LV GLS (\%) & $22.9 \pm 2.9^{\mathrm{a}}$ & $17.8 \pm 2.9^{b}$ & $20.3 \pm 2.3^{c}$ \\
\hline Transmitral E/A ratio & $0.93 \pm 0.4$ & $0.94 \pm 0.4$ & $1.0 \pm 0.3$ \\
\hline E velocity DT (ms) & $230.4 \pm 42.7$ & $206.6 \pm 41.5$ & $208.4 \pm 43.9$ \\
\hline E/e' ratio & $8.4 \pm 2.1$ & $9.1 \pm 2.9$ & $8.3 \pm 2.5$ \\
\hline LAVi $\left(\mathrm{mL} / \mathrm{m}^{2}\right)$ & $24.5 \pm 7.6$ & $29.1 \pm 9.9$ & $29.0 \pm 6.5$ \\
\hline
\end{tabular}

CTRCD, cancer therapy-related cardiotoxicity; DT, deceleration time; EC, epirubicine + cyclophoshamide; EF, ejection fraction; GLS, global longitudinal strain; LAVi, left atrial volume index; LV, left ventricular; TRZ, trastuzumab. ap $<0.0001$ baseline vs. CTRCD onset.

${ }^{b} P<0.004$ CTRCD onset vs. end of anticancer therapy.

${ }^{c} P=0.002$ baseline vs. end TRZ.

successfully demonstrated the possibility of identifying subclinical CTRCD by using troponin I. An early troponin I increase predicted subsequent EF reduction and outcomes (mainly HF) in patients undergoing anthracyclines or adjuvant therapy, thus addressing initiation of cardioprotective treatment with ACE inhibitors. ${ }^{18-22}$ This strategy is limited by the need of serial troponin I assessment and by the lack of a defined troponin I threshold for diagnosis of CTRCD onset. An alternative is currently represented by EF monitoring. In a recent study, the EF determination at baseline and every 3 months during $C T$ and the initiation of cardioprotection in case of overt CTRCD (=EF decrease $>10$ absolute points, and $<50 \%$ ), led to a full recovery of $L V$ systolic function ( $E F$ increase to baseline value) or at least to a partial recovery ( $E F$ increase $>5$ absolute points and $>50 \%$ ) in $71 \%$ of the patients. ${ }^{21}$ The main limitation of EF corresponds to its temporal (day-to-day) variability which is suboptimal (greater than $10 \%)^{9}$ and does not allow to attribute a definite clinical value to its changes.

In the present study, we applied the GLS strategy suggested by the ASE/EACVI Expert Consensus to identify subclinical CTRCD ${ }^{5}$ and timely started cardioprotection. The superior feasibility ${ }^{23}$ and reproducibility (inter-exam variability of about $6 \%$ ) of GLS in comparison with EF (about $10 \%)^{24}$ is well-known and allows a friendly use of this parameter in the oncologic setting. ${ }^{25}$ Moreover, LV longitudinal dysfunction testified by GLS precedes EF reduction, being particularly useful in the preclinical stages of several cardiac diseases, ${ }^{26,27}$ including CTRCD. ${ }^{28,29}$ Accordingly, the European Society of Cardiology has recently promoted the possibility of monitoring GLS changes during $\mathrm{CT}^{8}{ }^{8} \mathrm{~A}$ GLS drop $>15 \%$ from baseline is a concrete variation to predict HF in cancer patients undergoing concurrent TRZ and anthracycline therapy, independently on baseline EF. ${ }^{30}$ Changes of GLS appear to parallel the increase of troponin I values as an expression of myocardial cell damage, both being predictive of overt CTRCD in cancer patients. ${ }^{30}$ By embracing this concept, even when GLS remains widely normal, i.e. $>19 \%$, the occurrence of its drop of at least $15 \%$ during CT should be considered clinically relevant to identify a subclinical cardiac damage and start cardioprotective regimen. ${ }^{5,8}$

To the best of our knowledge, this is the first study to demonstrate that in a population of HER2-positive breast cancer patients undergoing CT the GLS-based approach and the consequent and timely initiation of cardioprotection with concomitant ACE-inhibitors and beta-blockers, may have a clear impact on the onset of subsequent overt HF and on CT withdrawn. A previous non-randomized observational study by Negishi et al. ${ }^{31}$ has similarities but also clear differences with our study. By applying a GLS decrease $\geq 11 \%$ to detect subclinical LV dysfunction and initiate cardioprotective therapy with beta-blockers at discretion of clinician, an improvement of both 


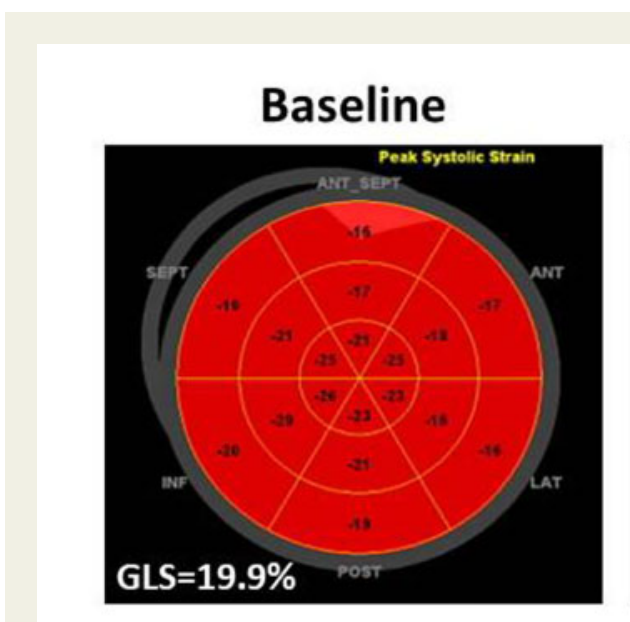

LV EF: $60 \%$

\section{Subclinical CTRCD}

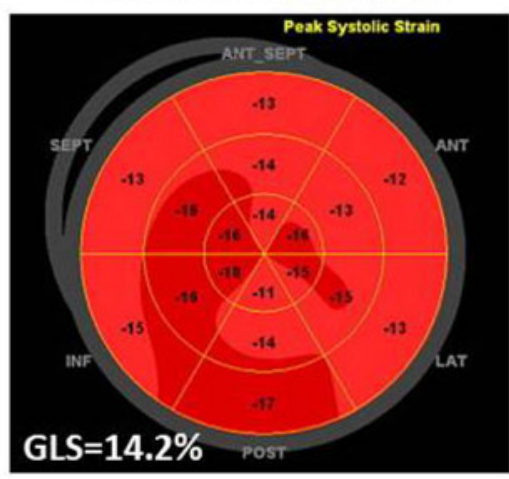

\section{TRZ completion}

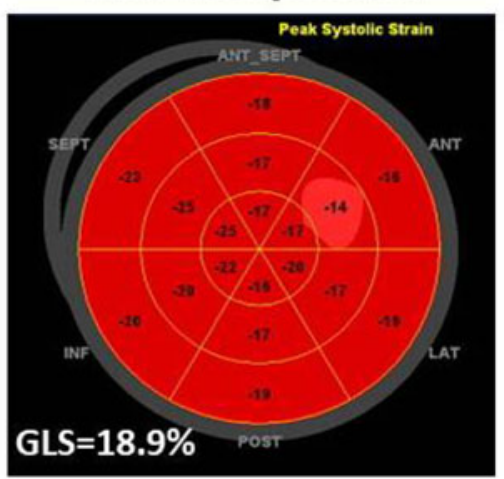

LV EF: $56 \%$

LV EF: $60 \%$

Figure 3 Clinical case of a patients developing subclinical CTRCD at the XI TRZ cycle but completing successfully TRZ thanks the timely cardioprotective treatment. Bull's eyes of GLS at baseline (left), at the time of subclinical CTRCD (mid), and at cancer therapy completion (right). CTRCD, cancer therapy-related cardiotoxicity; EF, ejection fraction; GLS, global longitudinal strain; TRZ, trastuzumab.

EF and GLS was found in the beta-blocker group but not in the non-beta-blocker group. In the present study, we used a GLS drop $>15 \%$ - as suggested by ASE and EACVI ${ }^{5}$ - and a combined cardioprotective regimen with beta-blockers and ACE-inhibitors.

It is noteworthy that, by applying this strategy, the incidence of overt CTRCD (3.4\%) was substantially lower than that reported in other recent investigations (from 7\% to 34\%). ${ }^{21,32}$ Most importantly, all the 23 patients developing subclinical CTRCD without progression towards overt HF were able to complete CT without interruption, also obtaining an almost complete recovery of both GLS and EF thanks to the cardioprotective regimen. The strain-oriented approach provided a positive impact even in the four patients (3.4\%) developing subsequent overt HF (overt CTRCD) since two out of four patients showed a full recovery (EF increase to baseline value), and one patient showed at least a partial recovery (EF increase $>5$ absolute points and $>50 \%$ ). The remaining patient, not showing any EF recovery and being symptomatic, was forced to permanently interrupt CT. Combined all together, these results demonstrate that the application of this innovative approach may allow to accomplish CT in almost the totality (96.5\%) of cancer patients.

An ancillary but important observation is also represented by the fact that in the remaining $76.7 \%$ of patients not developing subclinical or overt CTRCD, significant changes of both LV systolic (reduction of $\mathrm{EF}$ and GLS) and diastolic function (increase of $E / \mathrm{e}^{\prime}$ ratio) were detectable at the end of CT in comparison with baseline. This finding demonstrates a global detrimental cardiac effect of CT on LV function in the setting of breast cancer and highlights the need of careful monitoring LV function even after the CT completion.

\section{Study limitations}

The main limitation corresponds to the lack of a head-to-head comparison between 'standard LV EF' vs. 'GLS-based' guidance in addressing cardioprotective therapy in the cancer setting. Accordingly, the 'true' impact of strain-oriented cardiac therapy on GLS and LVEF recovery or on the withdrawal of chemotherapy cannot be definitively established by our findings. Moreover, similarly to EF, GLS is a load-dependent parameter and has a variability which prevents to attribute an absolute clinical value to its fluctuations during follow-up. Nevertheless, the day-to-day variability of GLS is much lower than the one presented by $\mathrm{EF}^{24}$ and, based on evidences, ${ }^{30}$ a GLS decrease $>15 \%$ from baseline is a cornerstone of ASE/ EACVI Expert Consensus on imaging evaluation of adult patients during and after $\mathrm{CT}^{5}$. The 'Strain Surveillance of Chemotherapy for Improving Cardiovascular Outcomes' (SUCCOUR) trial is an ongoing international multicentric prospective randomized controlled trial that aims to analyse the different aspects of the two approaches (GLS vs. EF) in the diagnosis and management of oncologic patients at high risk of cardiotoxicity. ${ }^{33}$ The results of this trial will probably provide definitive insights to put GLS in first line for surveillance of CTRCD. Another possible limitation corresponds to the absence of biomarker monitoring, very challenging to obtain in the outpatients referring to our University Hospital because of organizational procedures. Although its role remains controversial in this clinical setting, ${ }^{8}$ troponin I has been successfully used to detect subclinical CTRCD and predict subsequent development of overt HF. ${ }^{18-20}$ It could also have been very innovative to verify if GLS could anticipate the increase of NT-proBNP or BNP values compared to the behaviour 
of LVEF. However, the role of atrial peptides is not well-established for defining the routine surveillance of high-risk patients in the European position statement of cancer treatments and cardiovascular toxicity. ${ }^{8}$ Of note, BNP levels predicted overt CTRCD in some studies but not in others. ${ }^{30,34,35}$ Finally, the inter-vendor variability and technical requirements of GLS are intrinsic to the strain technology. ${ }^{8,28}$ Although recent studies have demonstrated a good concordance of GLS among different vendors, ${ }^{24}$ its measurement remains vendor-specific. ${ }^{36}$ Accordingly, serial assessment of GLS during CT should performed using the same vendor machine.

\section{Conclusion}

Our study puts a spotlight on the 'GLS-oriented' approach in detecting CTRCD and its prompt treatment, a matter that both cardiologists and oncologists will have to be aware of in the future years. This strategy may effectively prevent overt and irreversible HF and avoid the possible subsequent interruption of $\mathrm{CT}$, with clear negative reflections on cancer progression end relapse. Ongoing and future studies could open new horizons and possibly even define the lowest value of GLS to be considered as clinically relevant.

\section{Funding}

This work was supported by the International $\mathrm{PhD}$ Program in Cardiovascular Pathophysiology and Therapeutics CardioPath (to C.S., M.L., and R.S.).

\section{Conflict of interest: none declared.}

\section{References}

1. Siegel R, De Santis C, Virgo K, Stein K, Mariotto A, Smith T et al. Cancer treatment and survivorship statistics, 2012. CA Cancer J Clin 2012;62:220-41.

2. Ferlay J, Steliarova-Foucher E, Lortet-Tieulent J, Rosso S, Coebergh JW, Comber $\mathrm{H}$ et al. Cancer incidence and mortality patterns in Europe: estimates for 40 countries in 2012. Eur J Cancer 2013;49:1374-403.

3. Ewer MS, Ewer SM. Cardiotoxicity of anticancer treatments. Nat Rev Cardiol 2015;12:620.

4. Carver JR, Shapiro CL, Ng A, Jacobs L, Schwartz C, Virgo KS et al.; ASCO Cancer Survivorship Expert Panel. American Society of Clinical Oncology clinical evidence review on the ongoing care of adult cancer survivors: cardiac and pulmonary late effects. J Clin Oncol 2007;25:3991-4008.

5. Plana JC, Galderisi M, Barac A, Ewer MS, Ky B, Scherrer-Crosbie M et al. Expert consensus for multimodality imaging evaluation of adult patients during and after cancer therapy: a report from the American Society of Echocardiography and the European Association of Cardiovascular Imaging. Eur Heart J Cardiovasc Imaging 2014;15:1063-93.

6. Madeddu C, Deidda M, Piras A, Cadeddu C, Demurtas L, Puzzoni M et al. Pathophysiology of cardiotoxicity induced by nonanthracycline chemotherapy. J Cardiovasc Med 2016;17(Suppl 1):S12-18.

7. Herrmann J, Lerman A, Sandhu NP, Villarraga HR, Mulvagh SL, Kohli M. Evaluation and management of patients with heart disease and cancer: cardio-oncology. Mayo Clin Proc 2014;89:1287-306.

8. Zamorano JL, Lancellotti P, Rodriguez Muñoz D, Aboyans V, Asteggiano R, Galderisi M et al.; ESC Scientific Document Group. 2016 ESC Position Paper on cancer treatments and cardiovascular toxicity developed under the auspices of the ESC Committee for Practice Guidelines: the task force for cancer treatments and cardiovascular toxicity of the European Society of Cardiology (ESC). Eur Heart J 2016;37:2768-801.

9. Thavendiranathan P, Grant AD, Negishi T, Plana JC, Popović ZB, Marwick TH et al. Reproducibility of echocardiographic techniques for sequential assessment of left ventricular ejection fraction and volumes: application to patients undergoing cancer chemotherapy. J Am Coll Cardiol 2013;61:77-84.

10. Charbonnel C, Convers-Domart R, Rigaudeau S, Taksin AL, Baron N, Lambert J et al. Assessment of global longitudinal strain at low-dose anthracycline-based chemotherapy, for the prediction of subsequent cardiotoxicity. Eur Heart J Cardiovasc Imaging 2017;18:392-401.

11. Esposito R, Galderisi M, Santoro C, Imbriaco M, Riccio E, Pellegrino MA et al.; AFFINITY Group. Prominent longitudinal strain reduction of left ventricular basal segments in treatment-naïve Anderson-Fabry disease patients. Eur Heart J Cardiovasc Imaging 2019;20:438-45.

12. Lembo M, Esposito R, Lo ludice F, Santoro C, Izzo R, De Luca N et al. Impact of pulse pressure on left ventricular global longitudinal strain in normotensive and newly diagnosed, untreated hypertensive patients. J Hypertens 2016;34: 1201-7.

13. Lang RM, Badano LP, Mor-Avi V, Afilalo J, Armstrong A, Ernande L et al. Recommendations for cardiac chamber quantification by echocardiography in adults: an update from the American Society of Echocardiography and the European Association of Cardiovascular Imaging. Eur Heart J Cardiovasc Imaging 2015;15: 233-70.

14. Galderisi M, Cosyns B, Edvardsen T, Cardim N, Delgado V, Di Salvo G et al.; 2016-2018 EACVI Scientific Documents Committee; 2016-2018 EACVI Scientific Documents Committee. Standardization of adult transthoracic echocardiography reporting in agreement with recent chamber quantification, diastolic function, and heart valve disease recommendations: an expert consensus document of the European Association of Cardiovascular Imaging. Eur Heart J Cardiovasc Imaging 2017;18:1301-10.

15. de Simone G, Daniels SR, Devereux RB, Meyer RA, Roman MJ, de Divitiis O et al. Left ventricular mass and body size in normotensive children and adults: assessment of allometric relations and impact of overweight. J Am Coll Cardiol 1992; 20:251-60.

16. Nagueh SF, Smiseth OA, Appleton CP, Byrd BF 3rd, Dokainish H, Edvardsen T et al. Recommendations for the Evaluation of left ventricular diastolic function by Echocardiography: an update from the American Society of Echocardiography and the European Association of Cardiovascular Imaging. Eur Heart J Cardiovasc Imaging 2016;17:1321-60.

17. Alcidi GM, Esposito R, Evola V, Santoro C, Lembo M, Sorrentino R et al. Normal reference values of multilayer longitudinal strain according to age decades in a healthy population: a single-centre experience. Eur Heart J Cardiovasc Imaging 2018;19:1390-6.

18. Cardinale D, Sandri MT, Martinoni A, Tricca A, Civelli M, Lamantia G et al. Left ventricular dysfunction predicted by early troponin I release after high-dose chemotherapy. J Am Coll Cardiol 2000;36:517-22.

19. Cardinale D, Sandri MT, Martinoni A, Borghini E, Civelli M, Lamantia G et al. Myocardial injury revealed by plasma troponin I in breast cancer treated with high-dose chemotherapy. Ann Oncol 2002;13:710-5.

20. Cardinale D, Sandri MT, Colombo A, Colombo N, Boeri M, Lamantia G et al Prognostic value of troponin I in cardiac risk stratification of cancer patients undergoing high-dose chemotherapy. Circulation 2004;109:2749-54.

21. Cardinale D, Colombo A, Bacchiani G, Tedeschi I, Meroni CA, Veglia F et al. Early detection of anthracycline cardiotoxicity and improvement with heart failure therapy. Circulation 2015;131:1981-8.

22. Salvatici M, Cardinale D, Botteri E, Bagnardi V, Mauro C, Cassatella MC et al. Tnl-Ultra assay measurements in cancer patients: comparison with the conventional assay and clinical implication. Scand J Clin Lab Invest 2014;74: 385-91.

23. Santoro C, Arpino G, Esposito R, Lembo M, Paciolla I, Cardalesi C et al. 2D and $3 \mathrm{D}$ strain for detection of subclinical anthracycline cardiotoxicity in breast cancer patients: a balance with feasibility. Eur Heart J Cardiovasc Imaging 2017; 18:930-6.

24. Farsalinos KE, Daraban AM, Ünlü S, Thomas JD, Badano LP, Voigt JU. Head-tohead comparison of global longitudinal strain measurements among nine different vendors: the EACVI/ASE Inter-Vendor Comparison Study. J Am Soc Echocardiogr 2015;28:1171-81.

25. Bergler-Klein J. Strain and left ventricular volumes for predicting cardiotoxicity: a life-saving approach in anthracycline cancer treatment? Eur Heart J Cardiovasc Imaging 2015;16:968-9.

26. Galderisi M, Trimarco B. Global longitudinal strain: a novel hallmark of cardiac risk in arterial hypertension. J Hypertens 2016;34:1050-1.

27. Kalam K, Otahal P, Marwick TH. Prognostic implications of global LV dysfunction: a systematic review and meta-analysis of global longitudinal strain and ejection fraction. Heart 2014;100:1673-80.

28. Thavendiranathan P, Poulin F, Lim KD, Plana JC, Woo A, Marwick TH. Use of myocardial strain imaging by echocardiography for the early detection of cardiotoxicity in patients during and after cancer chemotherapy: a systematic review. J Am Coll Cardiol 2014;63:2751-68.

29. Negishi K, Negishi T, Hare JL, Haluska BA, Plana JC, Marwick TH. Independent and incremental value of deformation indices for prediction of trastuzumabinduced cardiotoxicity. J Am Soc Echocardiogr 2013;26:493-8. 
30. Sawaya H, Sebag IA, Plana JC, Januzzi JL, Ky B, Tan TC et al. Assessment of echocardiography and biomarkers for the extended prediction of cardiotoxicity in patients treated with anthracyclines, taxanes, and trastuzumab. Circ Cardiovasc Imaging 2012;5: 596-603.

31. Negishi K, Negishi T, Haluska BA, Hare JL, Plana JC, Marwick TH. Use of speckle strain to assess left ventricular responses to cardiotoxic chemotherapy and cardioprotection. Eur Heart J Cardiovasc Imaging 2014;15:324-31.

32. Moja L, Tagliabue L, Balduzzi S, Parmelli E, Pistotti V, Guarneri $\vee$ et al. Trastuzumab containing regimens for early breast cancer. Cochrane Database Syst Rev 2012;4:CD006243.

33. Negishi T, Thavendiranathan P, Negishi K, Marwick TH, Aakhus S, Murbræch K et al. Rationale and design of the strain surveillance of chemotherapy for improving cardiovascular outcomes (SUCCOUR) trial. JACC Cardiovasc Imaging 2018:11:1098-105.

34. Lenihan DJ, Massey MR, Baysinger KB, Adorno CL, Warneke CL, Steinert D et al. Superior detection of cardiotoxicity during chemotherapy using biomarkers. J Card Fail 2007;13:S151.

35. Witteles RM. Biomarkers as predictors of cardiac toxicity from targeted cancer therapies. J Card Fail 2016;22:459-64.

36. Voigt JU, Pedrizzetti G, Lysyansky P, Marwick TH, Houle H, Baumann R et al. Definitions for a common standard for 2D speckle tracking echocardiography: consensus document of the EACVI/ASE/Industry Task Force to standardize deformation imaging. Eur Heart J Cardiovasc Imaging 2015;16: $1-11$.

\title{
IMAGE FOCUS
}

\section{Aorta-right atrium tunnel: an unexpected diagnosis}

\author{
António Fontes $\mathbb{D}^{1}{ }^{1}$, Nuno Dias-Ferreira ${ }^{2}$, Ricardo Ladeiras-Lopes ${ }^{2}$, Marco Oliveira ${ }^{2}$, and Pedro Braga ${ }^{2}$ \\ ${ }^{1}$ Cardiology Department, Hospital do Divino Espırito Santo de Ponta Delgada, Avenida D. Manuel I, 9500-370 Ponta Delgada, Azores, Portugal; and ${ }^{2}$ Cardiology \\ Department, Centro Hospitalar Vila Nova de Gaia/Espinho, Rua Conceição Fernandes, 4434-502 Vila Nova de Gaia, Portugal \\ * Corresponding author. Tel: +351296203420; Fax: +351296203427. E-mail: antonioxfontes@hotmail.com
}

An asymptomatic 52-year-old woman was referred for the evaluation of a $4 / 6$ continuous murmur in the right parasternal border. Patient had no relevant family history of congenital heart disease. The electrocardiogram showed sinus rhythm and the echocardiogram revealed a round, vascular communication from the left aortic sinus (LAS) to the right atrium (RA), with continuous turbulent flow on Doppler evaluation. Additionally, a cardiac computed tomography angiography was performed for better delineation of coronary and extra-cardiac anatomy. 3D volume rendered reconstructions showed a large and tortuous 'tunnel-like' structure arising from the LAS, coursing posteriorly to the aortic root and terminating in the roof of the RA, just inferior and medial to the superior vena cava junction (Panel A). The left anterior descending (LAD) and the circumflex (LCX) arteries arose independently from the proximal portion of the tunnel. Cardiac magnetic resonance imaging with phase-contrast cine (PC-MRI) showed non-dilated right ventricle (RV), right ventricular outflow tract (RVOT) or main pulmonary artery (MPA) and there were no signs of pressure or volume overload (Panels B-E and Supplementary data online, Video S1). The Qp:Qs ratio assessed by PC-MRI was 1.8 (Panels F-l). The shunt volume assessed by in-plane PC-MRI was $37 \mathrm{~mL}$, and this result was comparable to the difference between the pulmonary (PA) and aortic (Ao) flow $(\mathrm{Qp}-\mathrm{Qs}=40 \mathrm{~mL})$. Once the patient was asymptomatic and there were no signs of right overload, we decided to manage the patient conservatively with close follow-up.

Aorta-RA tunnel (ARAT) is an extremely rare congenital anomaly. To the best of our knowledge, this is the oldest patient diagnosed with ARAT to be reported in the literature.

Supplementary data are available at European Heart Journal - Cardiovascular Imaging online.

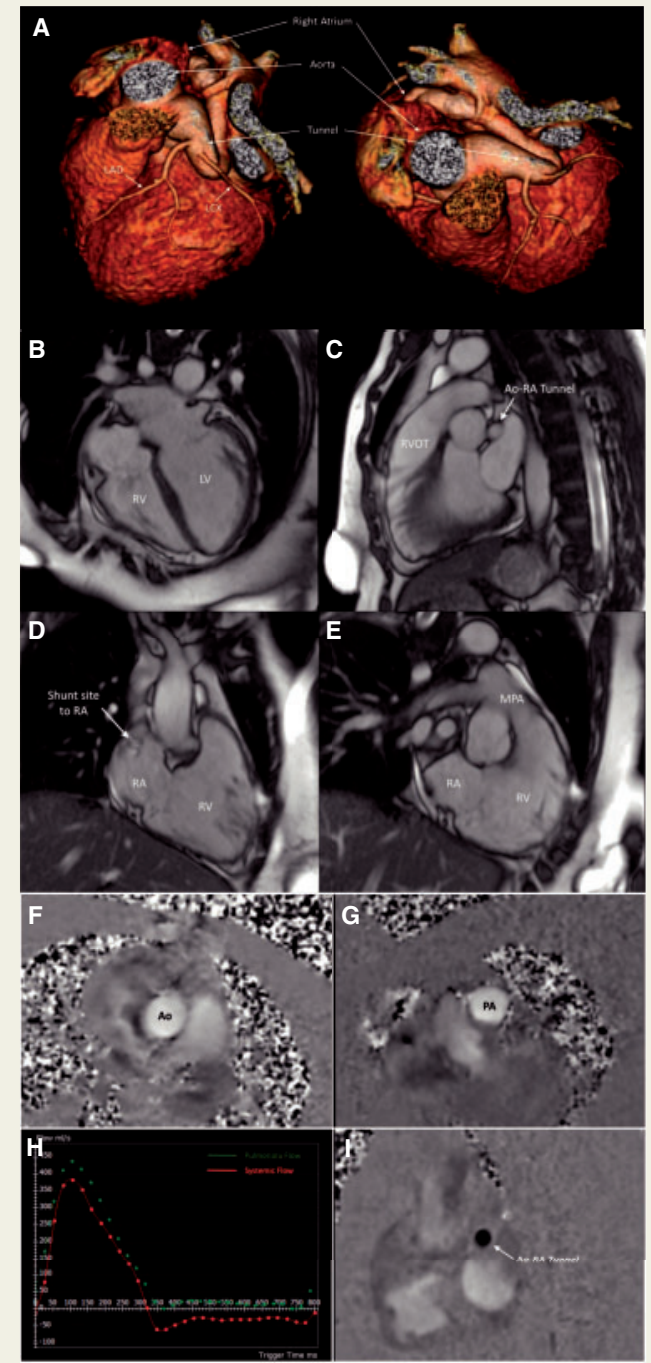

